\title{
Cognitive Polar Receiver Using Two Injection-Locked Oscillator Stages
}

\author{
Chi-Tsan Chen, Student Member, IEEE, Chieh-Hsun Hsiao, Student Member, IEEE, \\ Tzyy-Sheng Horng, Senior Member, IEEE, Kang-Chun Peng, Member, IEEE, and Chien-Jung Li, Member, IEEE
}

\begin{abstract}
A novel cognitive polar receiver that utilizes two injection-locked oscillator stages to extract the modulation envelope and phase components of a received nonconstant envelope modulation signal without using phase-locked-loop-based carrier recovery circuitry is presented. The paper begins with a theoretical analysis of injection locking and pulling phenomena based on the discrete-time computation approach, and then develops the principles of the proposed receiver. The implemented prototype can cover a sensing bandwidth of $140 \mathrm{MHz}$ at a central frequency of $2.43 \mathrm{GHz}$ and perform $\pi / 4$ differential quadrature phase-shift keying and quadrature phase-shift keying demodulation with the best error vector magnitudes of $6.6 \%$ and $7.9 \%$, respectively, both at a symbol rate of $2 \mathrm{Ms} / \mathrm{s}$. Due to its simplicity, the proposed receiver has great potential as an energy-efficient architecture with low complexity for short-range wireless communications.
\end{abstract}

Index Terms-Carrier recovery, cognitive radios, direct-conversion receiver, frequency demodulation, injection-locked oscillators (ILOs), polar receiver, spectrum sensing.

\section{INTRODUCTION}

$\mathbf{F}$ EW OF the people who enjoy the convenience of wireless communication ever realize the environmental impact of this technology. Energy consumption continues to increase with the explosive growth in the number of base stations and subscribers, increasing carbon emissions and accelerating global warming. Therefore, developing energy-efficient radio architectures to reduce the wasted power in wireless systems has become important in recent years and is one of the main features of the green radio technology, a research program proposed in recent years [1]. Today, the direct-conversion receiver is widely adopted in wireless systems because of its simplicity and low cost. However, coherent demodulation in modern modulation

Manuscript received July 06, 2011; revised October 02, 2011; accepted October 06, 2011. Date of publication November 18, 2011; date of current version December 14, 2011. This work was supported in part by the National Science Council, Taiwan, under Grant 97-2628-E-110-041-MY3, Grant 100-2221-E110-081-MY3, and Grant 100-2622-E-110-001-CC1, and by the Department of Industrial Technology, Taiwan, under Grant 99-EC-17-A-01-S1-104. This paper is an expanded paper from the IEEE International Microwave Symposium, Baltimore, MD, June 5-10, 2011.

C.-T. Chen, C.-H. Hsiao, and T.-S. Horng are with the Department of Electrical Engineering, National Sun Yat-Sen University, Kaohsiung 804, Taiwan (e-mail: D963010023@student.nsysu.edu.tw; jason@ee.nsysu.edu.tw).

K.-C. Peng is with the Department of Computer and Communication Engineering, National Kaohsiung First University of Science and Technology, Kaohsiung 811, Taiwan (e-mail: peterpkg@ccms.nkfust.edu.tw).

C.-J. Li is with the Department of Electronic Engineering, National Taipei University of Technology, Taipei 106, Taiwan (e-mail: chnjung@ntut.edu.tw).

Color versions of one or more of the figures in this paper are available online at http://ieeexplore.ieee.org.

Digital Object Identifier 10.1109/TMTT.2011.2172811 schemes, such as digital phase-shift keying (PSK), is required to ensure detection performance. It often relies on phase-locked loop (PLL)-based carrier recovery, which inevitably increases the total power consumed by the receiver [2]. Compared to a conventional synchronization loop, injection locking may provide an easier means of carrier recovery procedure with reduced circuit complexity and power consumption. In [3] and [4], a new binary phase-shift keying (BPSK) to amplitude-shift keying (ASK) converter was proposed. This BPSK demodulator combines the phase responses of two different second-harmonic injection-locked oscillators (ILOs) to phase changes of input signals to generate corresponding ASK patterns. In [5], a low-power BPSK receiver that directly extracts the modulation information from the dynamic phase response of the two BPSK signal injected oscillators has been presented. Recently, an injection-locked quadrature receiver (ILQR) has been studied [6]. It utilizes an ILO that has a locking range much smaller than the signal modulation bandwidth to recover the carrier signal for coherent quadrature demodulation of quadrature phase-shift keying (QPSK) and eight phase-shift keying (8PSK) modulated signals. However, the simulation results shown in [6] reveal that the ILO output signal, even with bandpass filtering, is severely deteriorated by the injected modulation signals, resulting in considerably distorted demodulation results.

This paper proposes a cognitive polar receiver that uses two ILO stages. Our previous paper [7] provided brief theoretical discussions on the proposed architecture and preliminary experimental results. The first ILO stage in the proposed architecture is for separating the envelope signal and the phase-modulated carrier from an input nonconstant envelope modulation signal, while the second ILO stage performs noncoherent demodulation of the phase-modulated carrier. The implemented receiver prototype is demonstrated with $\pi / 4$ DQPSK and QPSK signal demodulation. The elimination of PLL-based carrier recovery makes the proposed receiver energy efficient, and thus has great potential for short-range wireless communications. This paper substantially expands the earlier one [7] in two respects. First, it develops in detail the theory of the cognitive polar receiver. The newly extended theory also helps to simplify the architecture by eliminating a formerly required phase shifter. Second, the presented spectrum sensing ability of the receiver makes it suitable for cognitive radio applications.

This paper is organized as follows. The behavior of an ILO under nonconstant envelope modulation signal injections is described in Section II. Section III presents the proposed cognitive polar receiver and its operating principles. The simulated and the experimental results of the receiver are presented and 


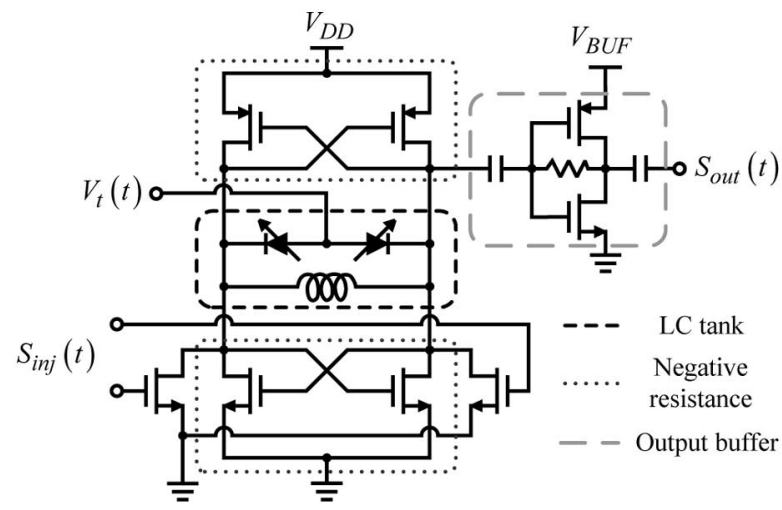

(a)

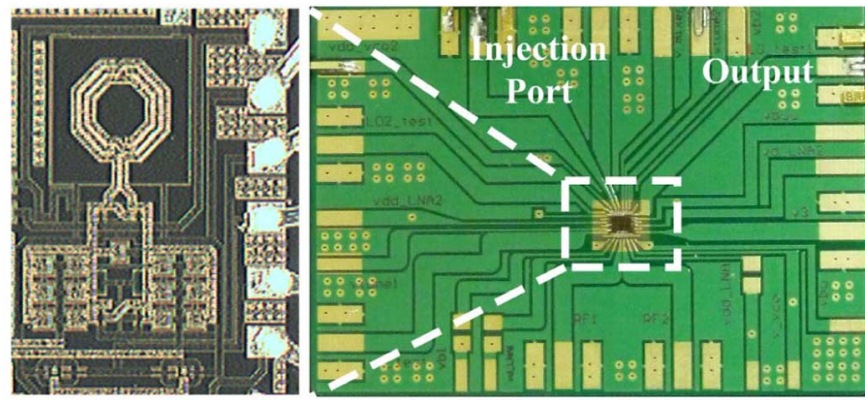

(b)

Fig. 1. (a) Circuit schematic of a VCO with an injection port. (b) Photographs of the implemented chip and its test board.

discussed in Section IV. A summary concludes this paper in Section V.

\section{GENERALIZED ChARACTERISTICS AND ANALYSIS OF ILOs}

Injection locking and pulling phenomena in oscillators have been extensively studied [8]-[15]. The classic analysis developed by Adler [8] considers that a weak sinusoidal signal interferes with an oscillator. His work elucidates the behavior of an ILO by deriving a differential equation known as Alder's equation. However, the analysis in this work is concerned with a general case in which the disturbance is a modulated signal with a time-varying envelope. Therefore, the mathematical model described in this section attempts to characterize an ILO under nonconstant envelope modulation signal injection. Modifications to Adler's equation are also made.

Fig. 1 depicts the simplified circuitry of the ILO and the photographs of the circuit in this work. A 2.36-GHz differential voltage-controlled oscillator (VCO) is designed at $1.2-\mathrm{V}$ supply voltage and fabricated in a $0.18-\mu \mathrm{m}$ CMOS process. The power consumptions of the VCO core and output buffer is 3 and $6 \mathrm{~mW}$, respectively. The VCO has a complementary cross-coupled pair to generate a negative resistance for oscillation and an inductance-capacitance $(L C)$ tank circuit, which has a quality factor of 6 , controlled by the tuning voltage $V_{t}(t)$. An attempt is made to increase the isolation between the injection port and the VCO output port, by injecting the injection signal $S_{\mathrm{inj}}(t)$ into the VCO core via another differential pair. The resultant VCO output signal under injection $S_{\text {out }}(t)$ is amplified by an output buffer. While taking $S_{\text {inj }}(t)$ as the refer- ence signal, Fig. 2(a) shows the vector relationship of signals in the ILO. The inherent oscillation signal $S_{\text {osc }}(t)$ has a constant amplitude $V_{\text {osc }}$ and an instantaneous frequency $\omega_{\text {osc }}(t) ; S_{\text {inj }}(t)$ has a time-varying amplitude $V_{\text {inj }}(t)$ and an instantaneous frequency $\omega_{\text {inj }}(t)$; and $S_{\text {out }}(t)$ has a constant amplitude $V_{\text {out }}$ and an instantaneous frequency $\omega_{\text {out }}(t)$. Since this paper considers a general case in which both the inherent oscillation signal $S_{\text {osc }}(t)$ and the injection signal $S_{\text {inj }}(t)$ are modulated signals, their instantaneous frequencies can be expressed as

$$
\begin{aligned}
& \omega_{\text {osc }}(t)=\omega_{\text {osc }}+\frac{d \theta_{o}(t)}{d t} \\
& \omega_{\text {inj }}(t)=\omega_{\text {inj }}+\frac{d \theta_{i}(t)}{d t}
\end{aligned}
$$

where $\omega_{\text {osc }}$ and $\omega_{\text {inj }}$ denote the center frequencies of $S_{\text {osc }}(t)$ and $S_{\text {inj }}(t)$, respectively; and $d \theta_{o}(t) / d t$ and $d \theta_{i}(t) / d t$ represent the instantaneous frequency modulations of $S_{\text {osc }}(t)$ and $S_{\text {inj }}(t)$, respectively. Notably, the instantaneous phase $\theta_{o}(t)$ can be regarded as the phase perturbation caused by phase noise, which is considered in the behavioral model of the ILO. Based on Adler's work [8], the relationship between the instantaneous frequencies of the resultant oscillator output signal under injection and the injection signal is given as

$$
\omega_{\text {out }}(t)=\omega_{\text {inj }}(t)+\frac{d \alpha(t)}{d t}
$$

where $\alpha(t)$ denotes the instantaneous angle between $S_{\text {osc }}(t)$ and $S_{\text {inj }}(t)$. The derivative of $\alpha(t)$ with respect to time represents a beat frequency between $\omega_{\text {out }}(t)$ and $\omega_{\text {inj }}(t)$, and it obeys the following generalized locking equation:

$$
\frac{d \alpha(t)}{d t}=-\omega_{\mathrm{LR}}(t) \beta(t)+\Delta \omega_{\mathrm{osc}}(t)
$$

where

$$
\begin{aligned}
\omega_{\mathrm{LR}}(t) & =\frac{\omega_{\mathrm{osc}}(t)}{2 Q} \frac{V_{\mathrm{inj}}(t)}{V_{\mathrm{osc}}} \\
\beta(t) & =\frac{\sin \alpha(t)}{1+\frac{V_{\mathrm{inj}}(t)}{V_{\mathrm{osc}}} \cos \alpha(t)} \\
\Delta \omega_{\mathrm{osc}}(t) & =\omega_{\mathrm{osc}}(t)-\omega_{\mathrm{inj}}(t) .
\end{aligned}
$$

$Q$ is the quality factor of a tank circuit. By adding $\omega_{\text {inj }}(t)$ on both sides of (4), the instantaneous frequency of the resultant oscillator output signal can also be expressed as

$$
\omega_{\text {out }}(t)=\omega_{\text {osc }}(t)-\omega_{\mathrm{LR}}(t) \beta(t) \text {. }
$$

Equation (8) provides the physical meaning that the instantaneous frequency of an oscillator under injection is shifted from its free-running frequency by an amount $\omega_{\mathrm{LR}}(t) \beta(t)$. In Fig. 2(a), $S_{\text {inj }}^{\prime}(t)$ is also an injection signal, but with a different amplitude from $S_{\mathrm{inj}}(t)$; in addition, the resultant oscillator output signal is $S_{\text {out }}^{\prime}(t)$. Fig. 2(b) illustrates the phase response of a simple tuned circuit [16]. The vector diagram clearly indicates that the angle between $S_{\text {osc }}(t)$ and $S_{\text {out }}(t)$, which is $\phi(t)$, moves to $\phi^{\prime}(t)$ when the injection signal changes from $S_{\text {inj }}(t)$ to $S_{\text {inj }}^{\prime}(t)$. Moreover, as shown in the phase response diagram, the corresponding change in frequency from $\omega_{\text {out }}(t)$ to $\omega_{\text {out }}^{\prime}(t)$ occurs. This mechanism is interpreted as an am- 


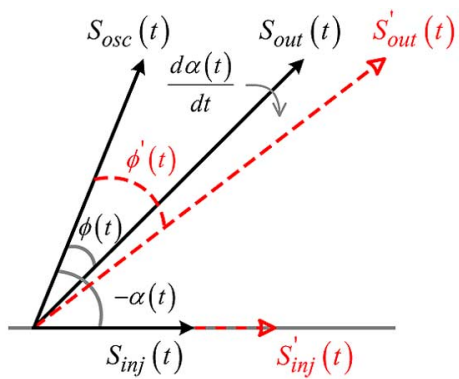

(a)

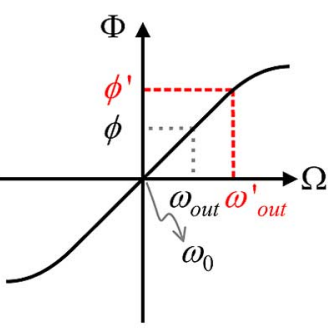

(b)
Fig. 2. Behavior of an oscillator under injection. (a) Vector diagram of the signals in the ILO. (b) Phase response of a simple tuned circuit.

plitude-modulation to frequency-modulation (AM-FM) effect caused by the time-varying amplitude injection signal. This signal induces frequency distortion of an ILO synchronized to the injected modulated signal.

Although the derived generalized locking (4) gives the phase dynamics of an oscillator under injection, gaining an analytical solution while considering a modulated injection signal is a rather complex task. Therefore, in this paper, performance of the proposed polar receiver is evaluated by using a discrete-time domain approach proposed in [15]. By starting with a numerical method known as the Euler method [17], the derivative can be replaced by the finite-difference approximation

$$
\frac{d \alpha(t)}{d t} \approx \frac{\alpha\left(t_{n}\right)-\alpha\left(t_{n-1}\right)}{\Delta t}
$$

where

$$
\Delta t=t_{n}-t_{n-1}
$$

Therefore, (4) is rewritten as

$$
\begin{aligned}
& \frac{\alpha\left(t_{n}\right)-\alpha\left(t_{n-1}\right)}{\Delta t} \\
& \approx-\omega_{\mathrm{LR}}\left(t_{n}\right) \beta\left(t_{n}\right)+\Delta \omega_{\mathrm{osc}}\left(t_{n}\right) \\
& \quad+\frac{\theta_{o}\left(t_{n}\right)-\theta_{o}\left(t_{n-1}\right)}{\Delta t}-\frac{\theta_{i}\left(t_{n}\right)-\theta_{i}\left(t_{n-1}\right)}{\Delta t}
\end{aligned}
$$

where

$$
\Delta \omega_{\text {osc }}\left(t_{n}\right)=\omega_{\text {osc }}\left(t_{n}\right)-\omega_{\text {inj }}
$$

A situation in which $\Delta t$ is sufficiently small makes it sensible to make an approximation that $\alpha\left(t_{n}\right)$ is equal to $\alpha\left(t_{n-1}\right)$. Replacing $\alpha\left(t_{n}\right)$ by $\alpha\left(t_{n-1}\right)$ in the right-hand side of (11) and rearranging lead to

$$
\begin{aligned}
\alpha\left(t_{n}\right)= & {\left[-\omega_{\mathrm{LR}}\left(t_{n}\right) \beta\left(t_{n}\right)+\Delta \omega_{\mathrm{osc}}\left(t_{n}\right)\right] \Delta t } \\
& +\theta_{o}\left(t_{n}\right)-\theta_{o}\left(t_{n-1}\right)-\theta_{i}\left(t_{n}\right) \\
& +\theta_{i}\left(t_{n-1}\right)+\alpha\left(t_{n-1}\right) \\
\omega_{\mathrm{LR}}\left(t_{n}\right)= & \frac{\omega_{\mathrm{osc}}\left(t_{n}\right)}{2 Q} \frac{V_{\mathrm{inj}}\left(t_{n}\right)}{V_{\mathrm{osc}}} \\
\beta\left(t_{n}\right)= & \frac{\sin \alpha\left(t_{n-1}\right)}{1+\frac{V_{\mathrm{inj}}\left(t_{n}\right)}{V_{\mathrm{osc}}} \cos \alpha\left(t_{n-1}\right)} .
\end{aligned}
$$

This approach is advantageous in that $\alpha\left(t_{n}\right)$ can be calculated recursively by (13) instead of solving differential equation (4);

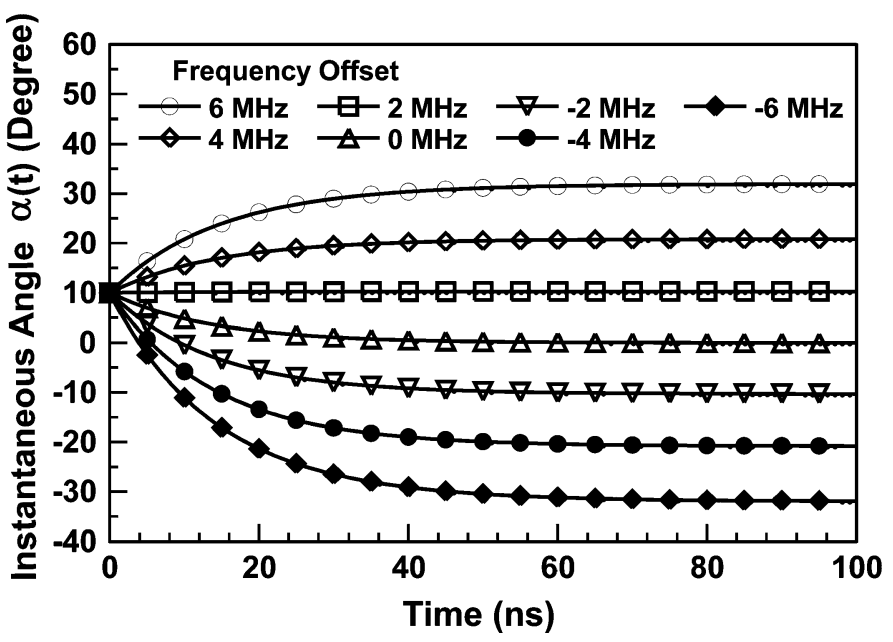

Fig. 3. Transient responses of $\alpha(t)$ at various frequency offsets $\Delta \omega_{\text {osc }}$ for an ILO under weak sinusoidal injection.

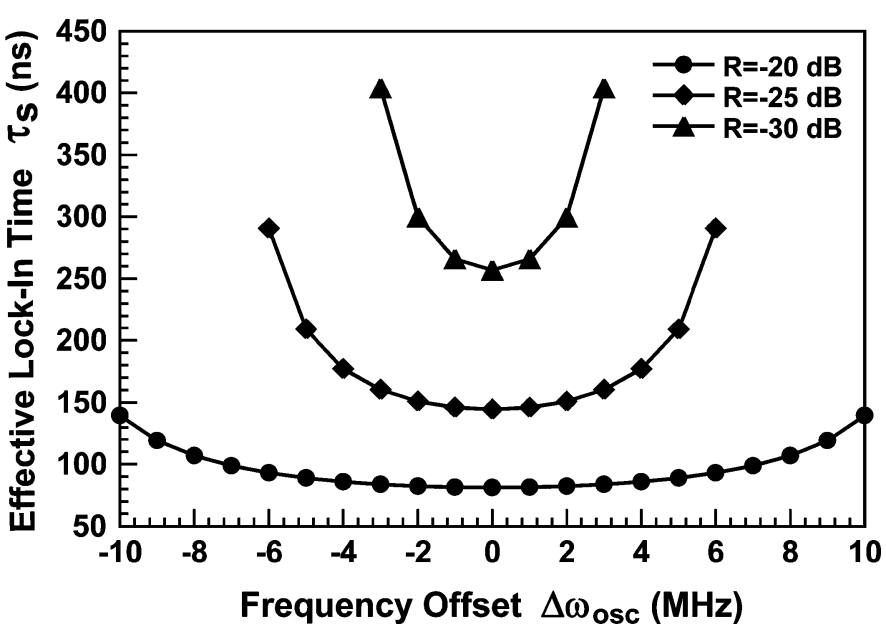

Fig. 4. Computed effective lock-in time of an ILO at various injection-to-oscillation voltage ratios $R$.

in addition, it is compatible with an arbitrary modulated injection signal. However, under some circumstances, approximating a derivative by the Euler method may not be sufficiently accurate. In this case, the computed results deviate from the measurement results when the power of the injection signal is comparable to the power of the inherent oscillation signal, i.e., under strong injection, as observed in Section IV. This inaccuracy arises from the drastic variation of phase $\alpha\left(t_{n}\right)$ under strong injection.

The transient time limits the maximum modulation rate of the injection signal that can synchronize an oscillator [10], [11], [18], [19]. Previous papers have derived a general solution of (4) for a weak sinusoidal injection signal [8], [11]. In the steady state, the phase difference between $S_{\text {inj }}(t)$ and $S_{\text {osc }}(t), \alpha(t)$, approaches a constant value $\alpha_{s s}$, which is related to an effective lock-in time of the ILO. Setting (4) equal to zero allows us to obtain the steady-state value of $\alpha(t)$ under weak sinusoidal injection as

$$
\alpha_{s s}=\sin ^{-1}\left(\frac{\Delta \omega_{\mathrm{osc}}}{\omega_{\mathrm{LR}}}\right)
$$




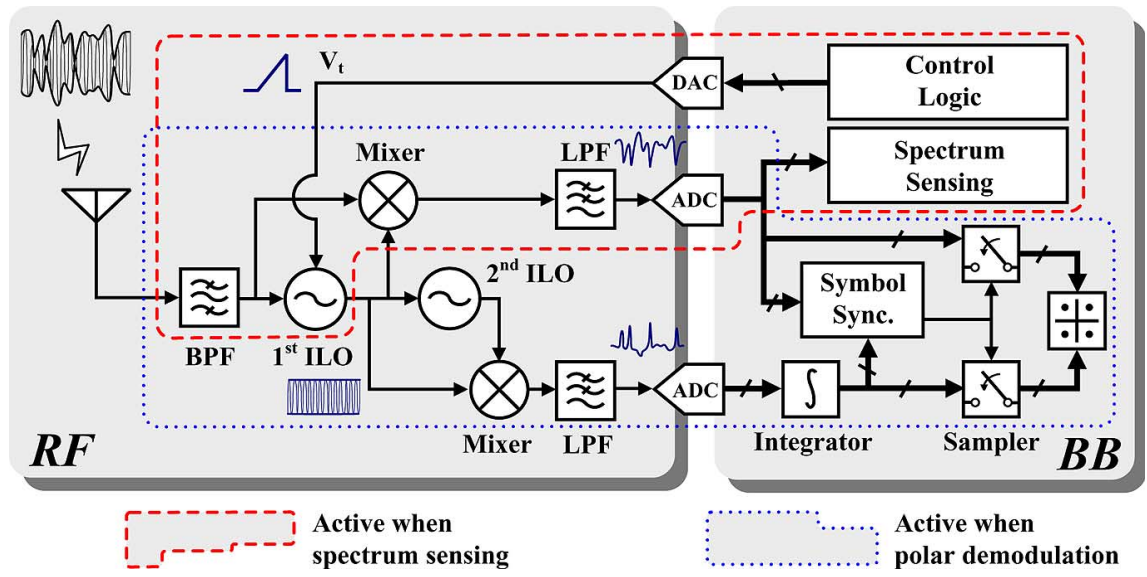

Fig. 5. Block diagram of the proposed cognitive polar receiver.

where

$$
\begin{aligned}
\omega_{\mathrm{LR}} & =\frac{\omega_{\mathrm{osc}}}{2 Q} \frac{V_{\mathrm{inj}}}{V_{\mathrm{osc}}} \\
\Delta \omega_{\mathrm{osc}} & =\omega_{\mathrm{osc}}-\omega_{\mathrm{inj}} .
\end{aligned}
$$

Fig. 3 plots the computed transient responses of the ILO under weak sinusoidal injection at various frequency offsets $\Delta \omega_{\text {osc }}$, where the constant steady-state values $\alpha_{s s}$ shown are described as (16). Notably, according to (16), $\alpha_{s s}$ depends on $\Delta \omega_{\text {osc }}$. Therefore, an effective lock-in time $\tau_{s}$ can be defined and derived as

$$
\tau_{s} \triangleq \frac{\partial\left(\alpha_{s s}\right)}{\partial\left(\Delta \omega_{\mathrm{osc}}\right)}=\frac{1}{\sqrt{\omega_{\mathrm{LR}}^{2}-\Delta \omega_{\mathrm{osc}}^{2}}} .
$$

Equation (19) reveals that an ILO can provide a variable time delay for use in a noncoherent frequency demodulator. Fig. 4 plots the computed effective lock-in time introduced by an ILO at various injection-to-oscillation voltage ratios, where the ratio is defined as

$$
R=20 \log \left(\frac{V_{\text {inj }}}{V_{\text {osc }}}\right) .
$$

The characterization and analysis approach of an ILO under nonconstant envelope modulation signal injection contribute to developing the operating principles of the cognitive polar receiver, which is described in Section III.

\section{RECEIVER ARCHITECTURE AND OPERATING PRINCIPLES}

\section{A. System Overview and Operation}

Fig. 5 shows the block diagram of the proposed cognitive polar receiver. This architecture comprises two parts: the RF section and the baseband section. The RF section is composed of ILOs, mixers, a bandpass filter (BPF), and low-pass filters (LPFs). In the baseband section, the digital signal processor (DSP) combined with analog-to-digital converters (ADCs) and a digital-to-analog converter (DAC) is responsible for digital control and demodulation.

The receiver is capable of spectrum sensing and polar demodulation. According to Fig. 5, the circuits encircled by the broken line are activated when the receiver operates in the spectrum sensing mode. First, the received signal is injected into the first ILO and mixed with the output signal of the first ILO. Meanwhile, the baseband control logic outputs frequency control words (FCWs) for the DAC to generate a ramp tuning voltage in order to sweep the inherent frequency of the first ILO in its tuning range. Detecting the low-pass filtered mixer output allows us to identify signal activities in this frequency range. Once a signal is identified for demodulation, the polar demodulation mode follows. The focus lies on the circuits encircled by the dotted line in Fig. 5. Next, the inherent frequency of the first ILO is now tuned to the center frequency of the received signal by the control logic. The first ILO stage operates in both the polar demodulation mode and the spectrum sensing mode. Additionally, the output signal of the first ILO is fed into the second ILO and mixed with the output signal of the second ILO. As revealed by (19) and Fig. 4, the effective lock-in time introduced of the ILO can be flexibly adjusted by varying the locking range $\omega_{\mathrm{LR}}$ or the frequency offset between the injection signal and the inherent oscillation signal $\left(\Delta \omega_{\text {osc }}\right)$. Therefore, the second ILO can be tuned to optimize the demodulation results. The first and second ILO stages extract the envelope modulation and the frequency modulation from the received signal, respectively. Furthermore, ADCs sample the output signals of both stages for the DSP to proceed to digital demodulation. Sections III-B and III-C illustrate in detail the mechanisms of the spectrum sensing and the polar demodulation.

\section{B. Spectrum Sensing}

Fig. 6 shows the small-signal block diagram of the RF section of the polar receiver, where the mixer is regarded as an ideal multiplier in the following derivations. Fig. 7 illustrates a conceptual diagram for demonstrating the spectrum sensing process. According to Fig. 7(a), the first ILO is swept by the tuning voltage $V_{t}(t)$ generated from the FCWs. Therefore, the inherent frequency of the oscillator changes continuously with

$$
\omega_{\mathrm{osc}}(t)=\omega_{\mathrm{osc}}+K_{v} V_{t}(t)
$$

where $\omega_{\text {osc }}$ is the center frequency and $K_{v}$ is the tuning sensitivity of the ILO. In Fig. 7(b), a signal $S_{\text {inj }}(t)$ is located in the tuning range of the ILO, $\omega_{L}$ to $\omega_{H}$. In the beginning of the 


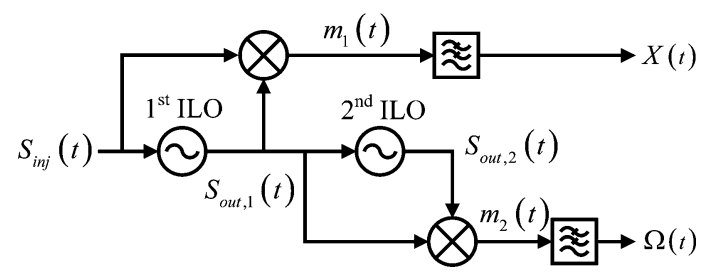

Fig. 6. Small-signal block diagram of the proposed cognitive polar receiver.

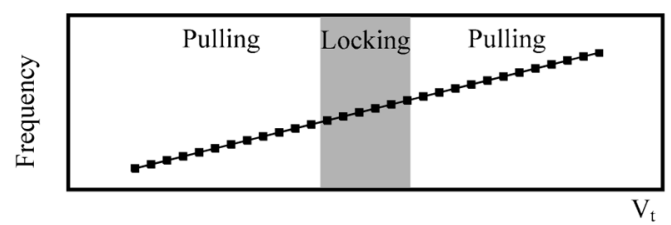

(a)

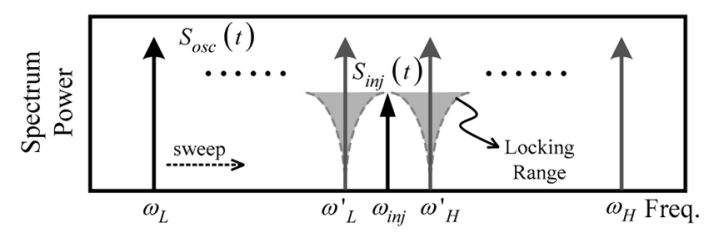

(b)

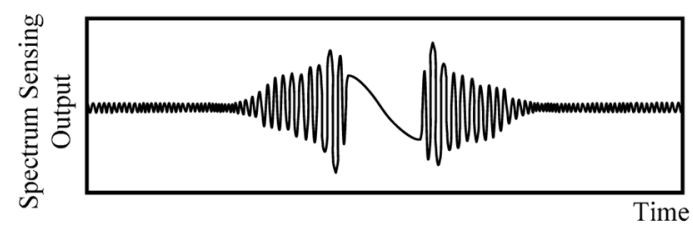

(c)

Fig. 7. Illustrations of the spectrum sensing mechanism. (a) Tuning voltage versus inherent frequency of the ILO. (b) Spectrum relationship of the inherent oscillation signal and the injection signal. (b) Output signal of the spectrum sensing circuits.

tuning period from frequency $\omega_{L}$ to $\omega_{L}^{\prime}$, the ILO experiences the injection-pulling condition since $S_{\text {osc }}(t)$ is too far from $S_{\text {inj }}(t)$ in the frequency to be locked. The instantaneous frequency of an oscillator $\omega_{\text {out }}(t)$ under the injection-pulling condition is frequency modulated with $d \alpha(t) / d t$, which has an average beat frequency $\omega_{b}(t)$ given as [13], [15]

$$
\omega_{b}(t)=\sqrt{\Delta \omega_{\mathrm{osc}}^{2}(t)-\left[\frac{\omega_{\mathrm{inj}}+\Delta \omega_{\mathrm{osc}}(t)}{2 Q \sqrt{1-\left(\frac{V_{\mathrm{inj}}}{V_{\mathrm{osc}}}\right.} \frac{V_{\mathrm{inj}}}{V_{\mathrm{osc}}}}\right]^{2}} .
$$

Notably, (22) is derived on the assumption that the frequency sweeping speed of the ILO, $70 \mathrm{MHz} / \mathrm{ms}$, is so fast that the modulated signal is a relatively narrowband one. Hence, the modulation envelope and phase can be viewed as time invariant in one single tuning period, i.e., $V_{\mathrm{inj}}(t) \approx V_{\mathrm{inj}}$ and $d \theta_{i}(t) / d t \approx 0$. Next, during the tuning period from frequency $\omega_{L}^{\prime}$ to $\omega_{H}^{\prime}$, the ILO is locked to the injection signal because $\omega_{\text {inj }}$ falls in the locking range of the ILO. By substituting (21) into (8), $\omega_{\text {out }}(t)$ can be further derived as

$$
\begin{aligned}
\omega_{\text {out }}(t) & =\omega_{\text {osc }}+K_{v} V_{t}(t)-\omega_{\mathrm{LR}}(t) \beta(t) \\
& \approx \omega_{\text {inj }}+K_{v} V_{t}(t) .
\end{aligned}
$$

Finally, the ILO is under injection pulling again when the inherent frequency is swept from $\omega_{H}^{\prime}$ to $\omega_{H}$.

According to Fig. 6, the mixer output of the first ILO stage is found as

$$
\begin{aligned}
m_{1}(t) & =S_{\mathrm{inj}}(t) \cdot S_{\mathrm{out}, 1}(t) \\
& \approx V_{\mathrm{inj}} \cos \left[\omega_{\mathrm{inj}} \cdot t\right] \cdot V_{\mathrm{osc}, 1} \cos \left[\omega_{\mathrm{out}, 1}(t) \cdot t\right] .
\end{aligned}
$$

Obviously, $m_{1}(t)$ contains the FM sidebands of the ILO when it is under injection pulling. Therefore, an LPF is utilized to filter out most of the sidebands, and pass the signal around dc. However, there is still a transition close to the region of injection locking. This is owing to that the closer that $\omega_{\text {osc }}(t)$ approaches $\omega_{\text {inj }}$ implies a lower beat frequency. If the injection-pulled FM sidebands fall into the passband of the filter, signal variations are detected, as the ripples demonstrated in Fig. 7(c). While the ILO is injection locked to $S_{\mathrm{inj}}(t),(23)$ indicates that $\omega_{\text {out }, 1}(t)$ is close to $\omega_{\text {inj }}$ and varies proportionally to the tuning voltage $V_{t}(t)$. Therefore, (24) can be further derived as

$$
\begin{aligned}
m_{1}(t) \approx & V_{\text {inj }} \cos \left[\omega_{\text {inj }} t\right] \\
& \cdot V_{\text {osc }, 1} \cos \left\{\left[\omega_{\text {inj }}+K_{v} V_{t}\left(t-\tau_{s, 1}\right)\right]\left(t-\tau_{s, 1}\right)\right\}
\end{aligned}
$$

where $\tau_{s, 1}$ denotes the effective lock-in time introduced by the first ILO. As computed in Section II, $\tau_{s, 1}$ is a short time compared to the frequency tuning period. Additionally, the phase shift corresponding to this lock-in time is zero at the center frequency of the injection signal due to the zero frequency offset, i.e., $\Delta \omega_{\text {osc }}=0$. Therefore, $X(t)$ is represented as

$$
X(t) \approx \frac{1}{2} V_{\mathrm{inj}} V_{\mathrm{osc}, 1} \cos \left[K_{v} V_{t}(t) t\right]
$$

which corresponds to the quasi-linear waveform in Fig. 7(c). Equation (26) suggests that the detected output $x(t)$ under injection locking correlates with the received signal level $V_{\text {inj }}$ and the tuning voltage $V_{t}(t)$. With the knowledge of the detected magnitude and the tuning voltage, the baseband spectrum sensing unit can determine the power and the frequency of the received signal.

\section{Polar Demodulation}

In the polar demodulation, both ILOs are assumed to be synchronized with the injected modulated signal. Therefore, the instantaneous frequency of the resultant output signal follows the frequency variations of the modulation and (3) can be rewritten as

$$
\omega_{\text {out }}(t) \approx \omega_{\text {inj }}\left(t-\tau_{s}\right)
$$

where $\tau_{s}$ is the effective lock-in time. Referring to Fig. 6, the mixer output of the first ILO stage $m_{1}(t)$ can be expressed as

$$
\begin{aligned}
m_{1}(t)= & S_{\mathrm{inj}}(t) \cdot S_{\mathrm{out}, 1}(t) \\
= & V_{\mathrm{inj}}(t) \cos \left[\omega_{\mathrm{inj}}(t) \cdot t\right] \\
& \cdot V_{\mathrm{osc}, 1} \cos \left[\omega_{\mathrm{inj}}\left(t-\tau_{s, 1}\right) \cdot\left(t-\tau_{s, 1}\right)\right]
\end{aligned}
$$


Here, the zero phase shift condition is applied again, as described in Section III-B, i.e.,

$$
\omega_{\text {inj }} \cdot \tau_{s, 1}=n \cdot 360^{\circ}, \quad n=0,1,2 \ldots
$$

As mentioned earlier, $\tau_{s, 1}$ is small. Therefore, $X(t)$ is found as

$$
\begin{aligned}
X(t) & \approx \frac{1}{2} V_{\mathrm{inj}}(t) V_{\mathrm{osc}, 1} \cos \left[\tau_{s, 1} \cdot \frac{d \theta_{i}\left(t-\tau_{s, 1}\right)}{d t}\right] \\
& \approx \frac{1}{2} V_{\mathrm{osc}, 1} V_{\mathrm{inj}}(t) .
\end{aligned}
$$

Analogously, the mixer output of the second ILO stage $m_{2}(t)$ is given as

$$
\begin{aligned}
m_{2}(t)= & S_{\text {out }, 1}(t) \cdot S_{\text {out }, 2}(t) \\
= & V_{\text {osc }, 1} \cos \left[\omega_{\text {out }, 1}(t) \cdot t\right] \\
& \cdot V_{\text {osc }, 2} \cos \left[\omega_{\text {out }, 2}(t) \cdot t\right] .
\end{aligned}
$$

As described in Section II, the equivalent lock-in time is adjustable by tuning the inherent frequency of the ILO. For the optimum frequency demodulation results, the second ILO is tuned to make the following condition valid:

$$
\omega_{\text {inj }} \cdot \tau_{s, 2}=90^{\circ}+n \cdot 360^{\circ}, \quad n=0,1,2, \ldots
$$

where $\tau_{s, 2}$ is the effective lock-in time of the second ILO. Therefore, $\Omega(t)$ is found as

$$
\begin{aligned}
\Omega(t)= & \frac{1}{2} V_{\mathrm{osc}, 1} V_{\mathrm{osc}, 2}(t) \\
& \cdot \cos \left[\omega_{\mathrm{inj}} \cdot \tau_{s, 2}+\tau_{s, 2} \cdot \frac{d \theta_{i}\left(t-\tau_{s, 2}\right)}{d t}\right] \\
\approx & \frac{1}{2} V_{\mathrm{osc}, 1} V_{\mathrm{osc}, 2} \cdot \tau_{s, 2} \cdot \frac{d \theta_{i}(t)}{d t} .
\end{aligned}
$$

Equation (30) and (33) represent the envelope modulation and the frequency modulation extracted from the received signal in the first ILO stage and the second ILO stage, respectively. The ADCs will sample both signals for digital signal processing. Based on the use of the digital integrator, the phase modulation is recovered from the measured frequency modulation, and baseband symbol synchronization is conducted to determine the correct sampling time [20].

\section{SimUlated AND EXPERIMENTAL RESUltS}

To verify the feasibility of the novel cognitive polar receiver, simulations and experiments are carried out. The discrete-time model is used to predict the performance of the polar demodulation. The implemented prototype of the receiver comprises two differential VCO integrated circuits with an output power of $-1.6 \mathrm{dBm}$ and other commercial discrete components, including LPFs with 39-MHz cutoff frequency and passive mixers with 8 -dB conversion loss. The ramp tuning voltage is generated by an arbitrary waveform generator (AWG). The dual-channel data acquisition (DAQ) samples the demodulated waveforms for

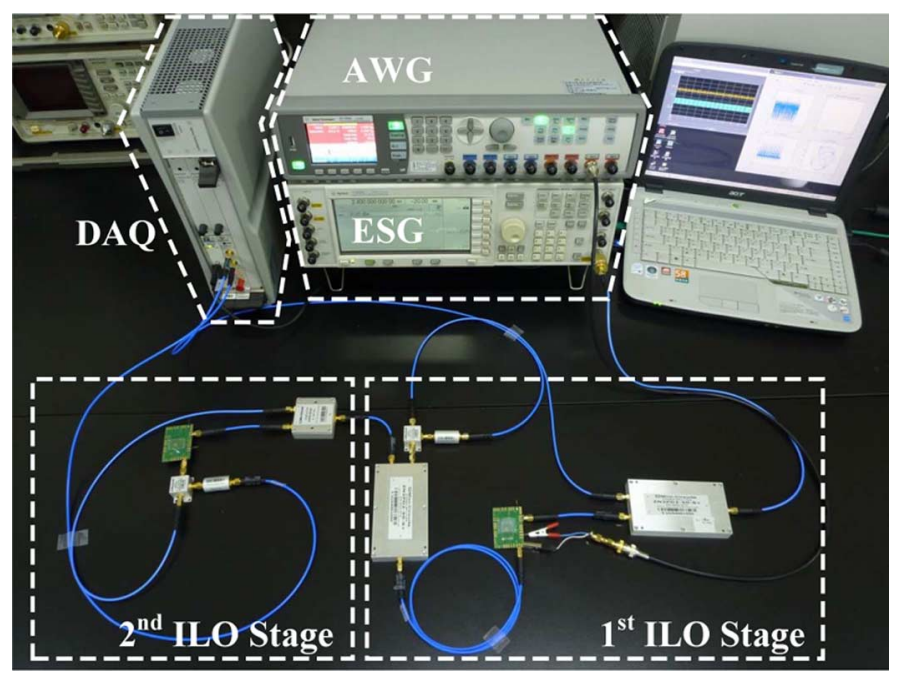

Fig. 8. Photograph of the test setup of the polar receiver.

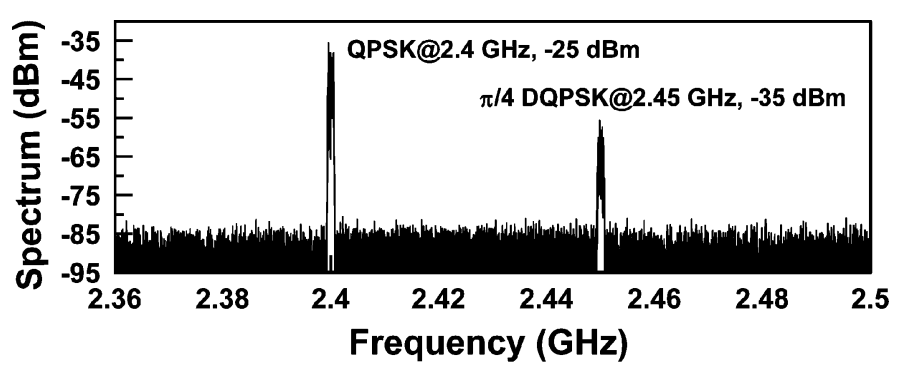

(a)

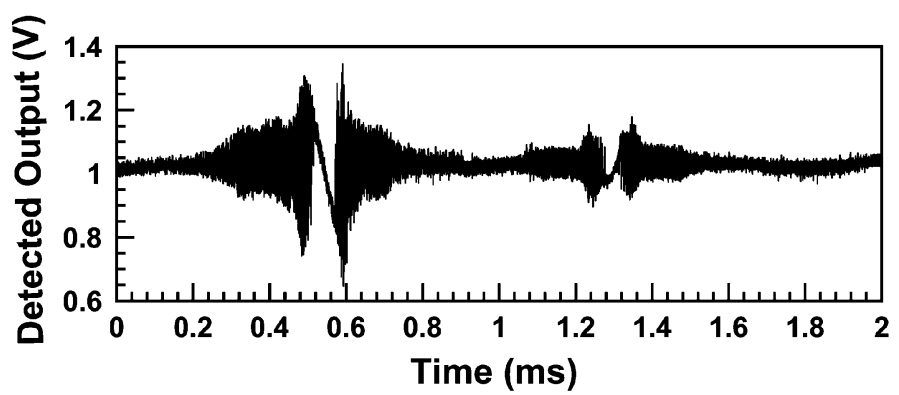

(b)

Fig. 9. Experiment of the spectrum sensing. (a) Spectrum of the combined test signal. (b) Measured output signal of the spectrum sensing circuit.

signal quality analysis. The baseband DSP functions are implemented in MATLAB language on a computer. Fig. 8 is a photograph of the receiver system.

In the spectrum sensing experiments, the inherent frequency of the VCO is swept from 2.36 to $2.5 \mathrm{GHz}$ and the ramp tuning voltage is varied linearly from 0 to $3.3 \mathrm{~V}$ in $2 \mathrm{~ms}$, leading to a $70-\mathrm{MHz} / \mathrm{ms}$ tuning rate. To test the spectrum-sensing functionality, two independent modulation signals are generated, combined, and sensed. Fig. 9(a) displays the spectrum of the combined signal. The first is a QPSK-modulated signal at $2.4 \mathrm{GHz}$ with a power of $-25 \mathrm{dBm}$ and the other is a $\pi / 4$ DQPSK-modulated signal at $2.45 \mathrm{GHz}$ with a power of $-35 \mathrm{dBm}$. Both signals have a 2-Ms/s data rate. As shown in Fig. 9(b), the two active regions correspond to two modulation signals. According 


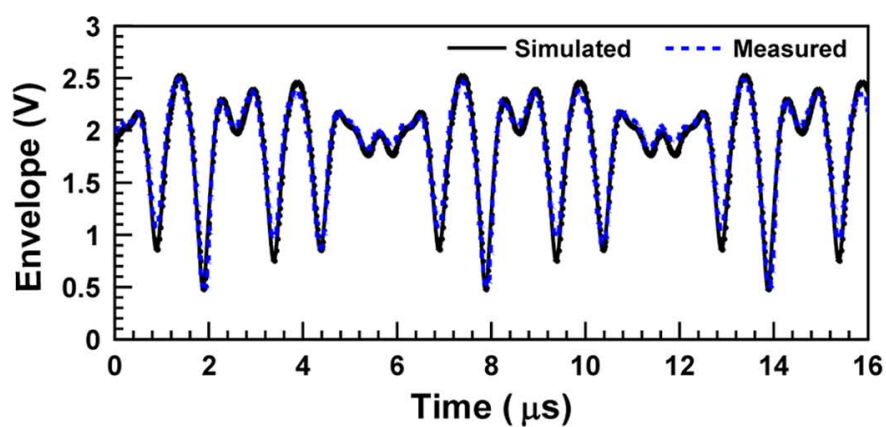

(a)

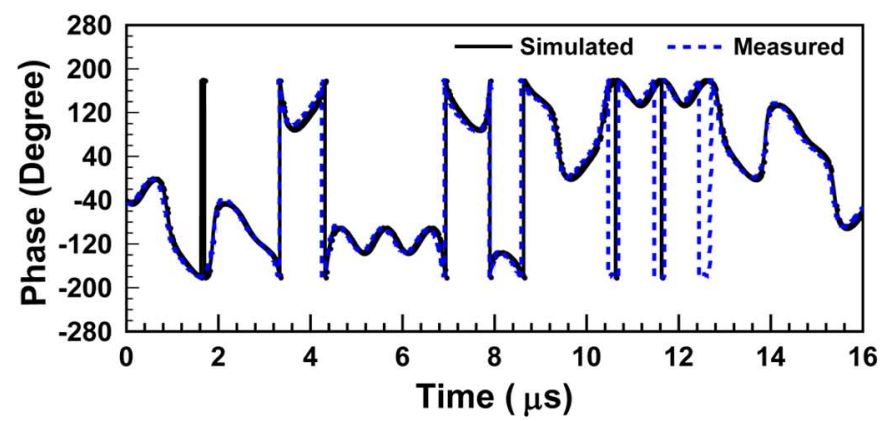

(b)

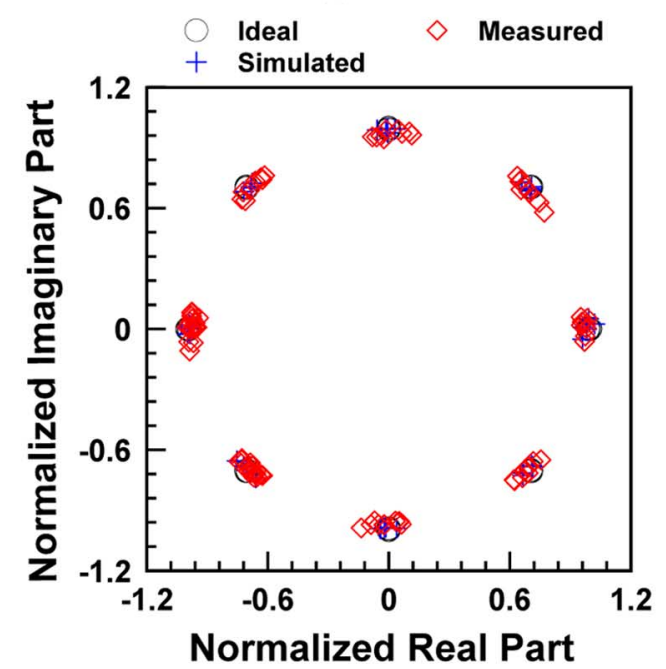

(c)

Fig. 10. Demodulated results for $\pi / 4$ DQPSK at $2 \mathrm{Ms} / \mathrm{s}$ and a received power of $-35-\mathrm{dBm}$ injected power. (a) Envelope. (b) Phase. (c) Constellation diagram.

to the measured results, the estimated frequency and power of the first signal are $2.395 \mathrm{GHz}$ and $-21.6 \mathrm{dBm}$, and those of the second are $2.457 \mathrm{GHz}$ and $-29.5 \mathrm{dBm}$. Notably, noise has a major influence on the accuracy of the spectrum sensing mechanism. However, this prototype serves as a proof of concept and reveals that the proposed architecture is suitable for cognitive radio applications.

The simulated and measured results of the polar demodulation follow. Fig. 10 displays the results of demodulating a $\pi / 4$ DQPSK-modulated signal, including the demodulated envelope and phase waveforms, as well as the corresponding constellation diagram at $2 \mathrm{Ms} / \mathrm{s}$ and a received power of $-35 \mathrm{dBm}$. The resultant error vector magnitude (EVM) from the measurements is $6.6 \%$ and that from the simulation results is $5.12 \%$. In

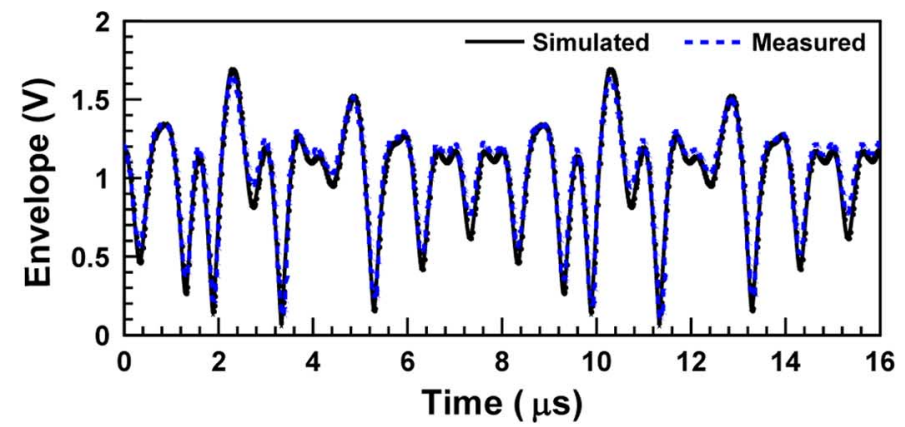

(a)

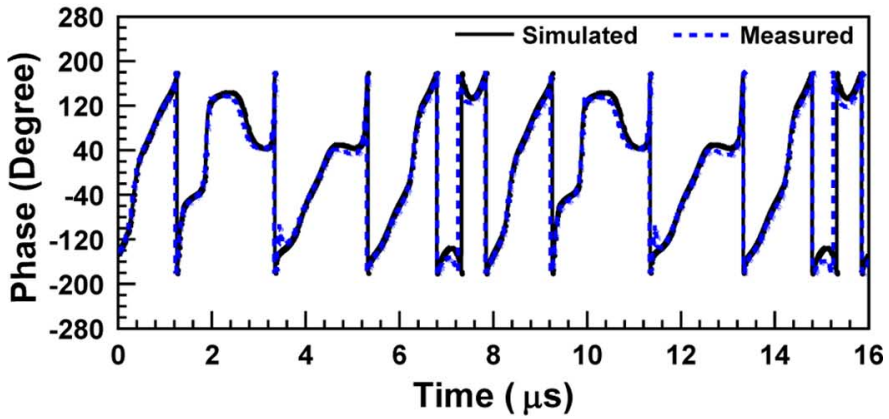

(b)

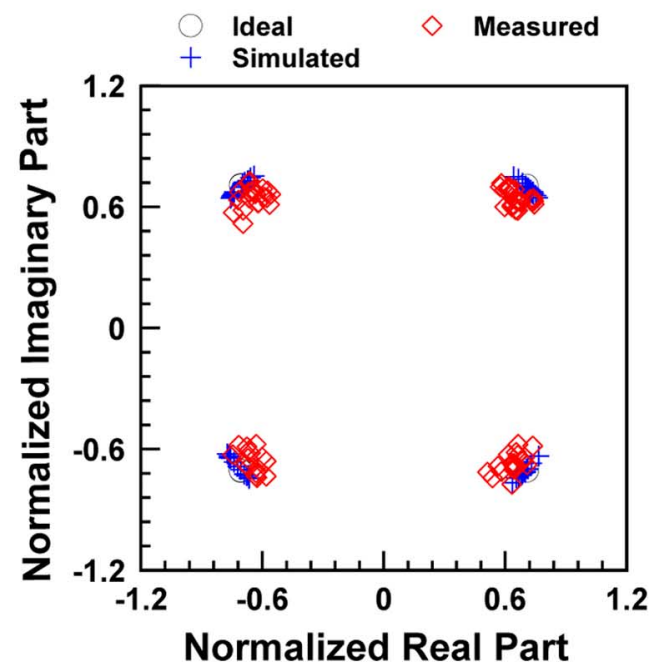

(c)

Fig. 11. Demodulated results for QPSK at $2 \mathrm{Ms} / \mathrm{s}$ and a received power of $-25-\mathrm{dBm}$ injected power. (a) Envelope. (b) Phase. (c) Constellation diagram.

a similar fashion, Fig. 11 displays the results of demodulating a QPSK-modulated signal at $2 \mathrm{Ms} / \mathrm{s}$ and a received power of $-25 \mathrm{dBm}$. The EVM from the measurements is $7.9 \%$ and that from the simulation results is $7.05 \%$, respectively.

The time mismatch between the envelope and phase is an important issue concerning conventional polar transmitters [21], and is also examined herein. Fig. 12 shows the measured and simulated EVM versus the delay difference between the two paths. These results evidence that the time mismatch is critical to the quality of demodulation in the polar receiver, and must be compensated for properly. This compensation can be performed by adding digital delays into the baseband to tune out the time mismatch. 


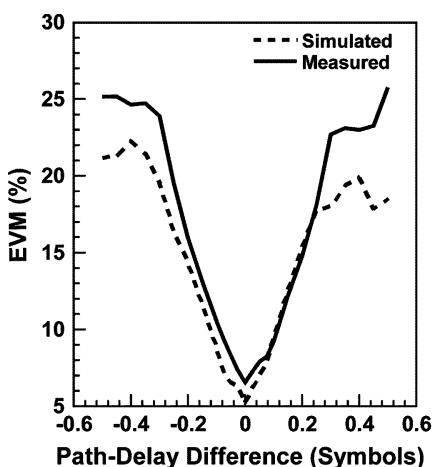

(a)

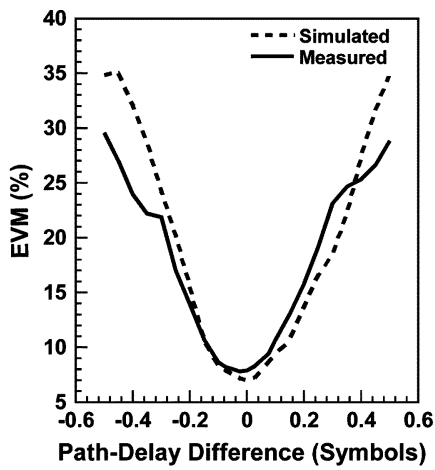

(b)
Fig. 12. Time mismatch effects on EVM. (a) $\pi / 4$ DQPSK at $2 \mathrm{Ms} / \mathrm{s}$ and a received power of $-35-\mathrm{dBm}$ injected power. (b) QPSK at $2 \mathrm{Ms} / \mathrm{s}$ and a received power of $-25-\mathrm{dBm}$ injected power.

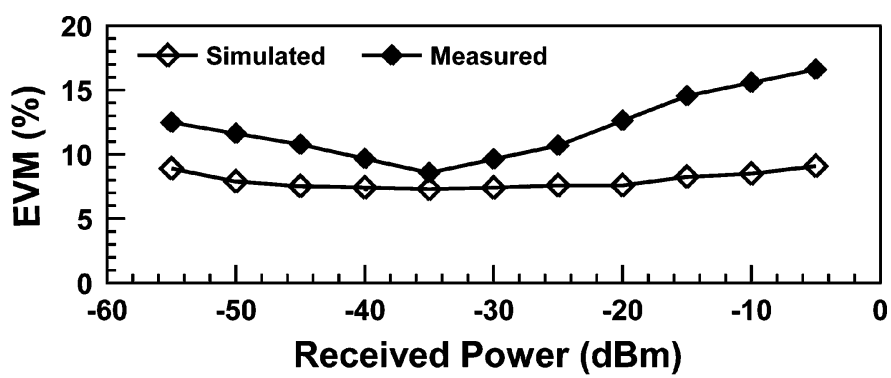

(a)

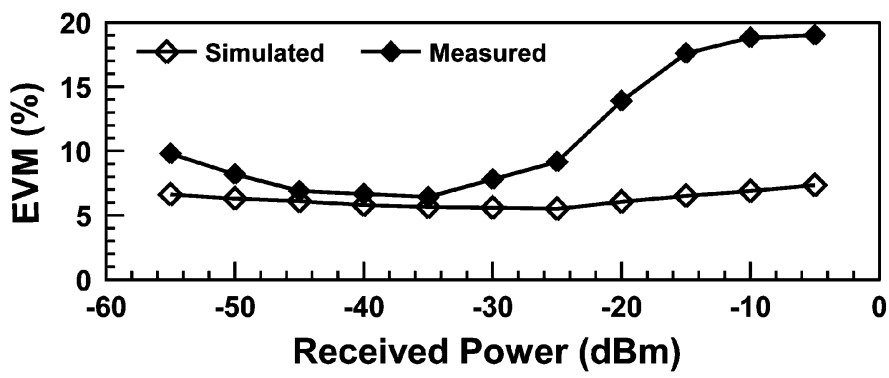

(b)

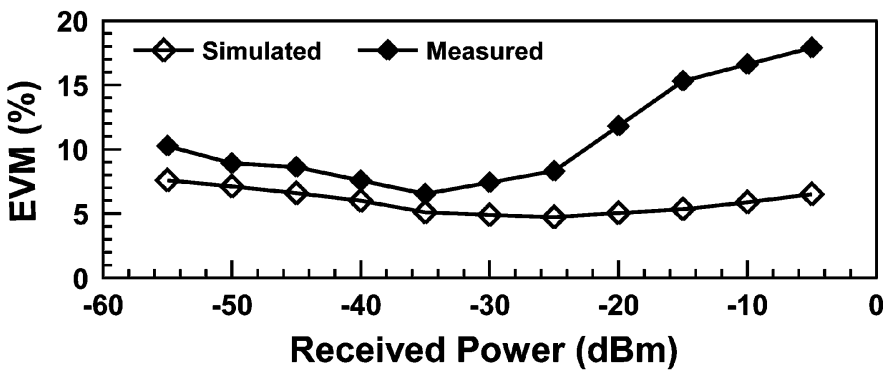

(c)

Fig. 13. EVM versus received power for $\pi / 4$ DQPSK. (a) $500 \mathrm{ks} / \mathrm{s}$. (b) $1 \mathrm{Ms} / \mathrm{s}$. (c) $2 \mathrm{Ms} / \mathrm{s}$

To evaluate the performance of the proposed receiver over its dynamic range, Figs. 13 and 14 plot the EVMs as a function of the received power at various symbol rates. Both the simulations and experiments reveal two main distortion mechanisms in the polar receiver. The first one is the AM-FM effect, which debases the reproduction of FM in a synchronized oscillator, meaning that the FM of the injected signal was distorted

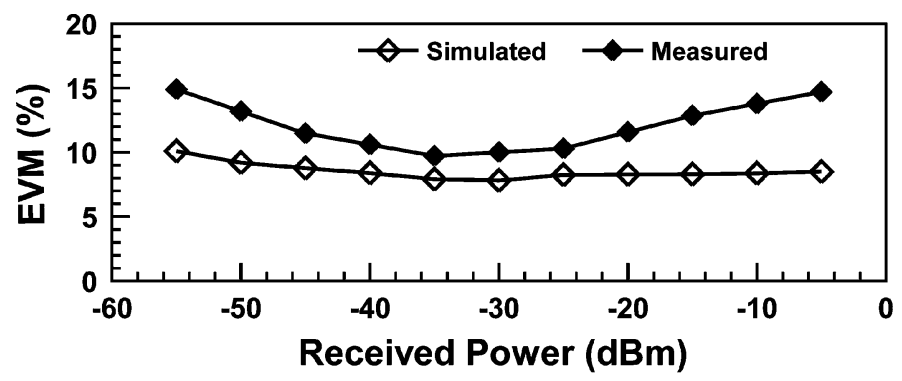

(a)

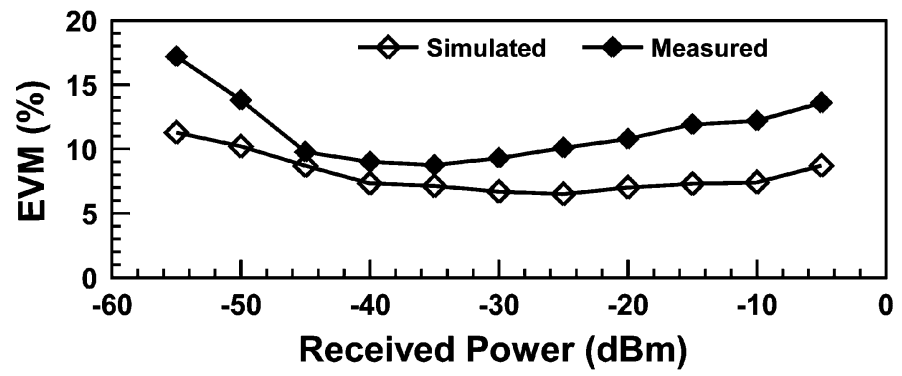

(b)

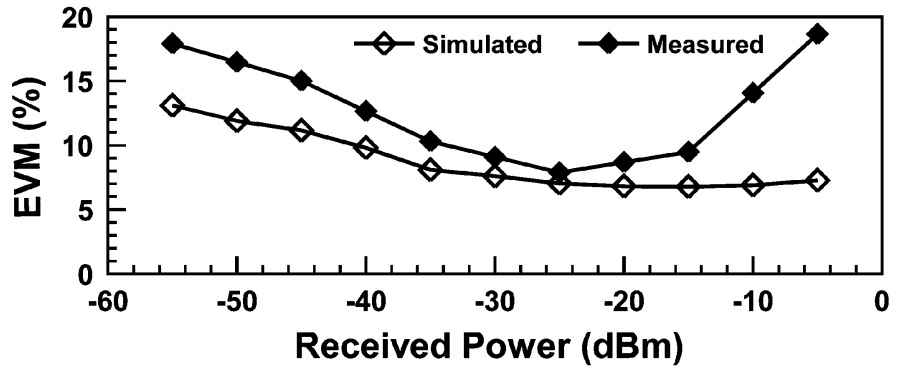

(c)

Fig. 14. EVM versus received power for QPSK. (a) $500 \mathrm{ks} / \mathrm{s}$. (b) $1 \mathrm{Ms} / \mathrm{s}$. (c) $2 \mathrm{Ms} / \mathrm{s}$.

in the first ILO stage, and therefore the EVM was degraded. The second one is the relationship between the effective lock-in time in (19) and the frequency offset $\Delta \omega_{\text {osc }}$ such that the $\tau_{s}$ of an ILO varies continuously with the injected FM signal from the first ILO. However, a constant delay must be maintained in the conventional noncoherent frequency demodulator. The replacement of a constant delay element with an ILO in the demodulator degrades the demodulation results. Both distortion mechanisms together determine the demodulation quality of the polar receiver. The demodulation results are generally better for $\pi / 4$ DQPSK than for QPSK because the QPSK-modulated signal has a higher peak-to-average power ratio (PAPR) than the $\pi / 4$ DQPSK-modulated signal. Hence, a higher AM-FM distortion is induced, degrading the demodulation results more. A few discrepancies are observed between the simulated and measured results around the lowest and the highest received powers in Figs. 13 and 14. On the low-power side, a discrepancy arises from the simplification of the simulation of the polar receiver to reduce the computation time. All sub-circuits, except for the ILOs, in the polar receiver are regarded as exhibiting ideal behavior. However, the noise of the mixer deteriorates the demodulated envelope signal in the first ILO stage. The experiment revealed obvious degradation of EVM. On the high-power side, however, since the discrete-time domain approach is used, the 
TABLE I

COMPARISON OF RECENT ILO-BASED RECEIVERS

\begin{tabular}{|c|c|c|c|c|c|c|}
\hline Reference & Technology & Frequency & Modulation & Data rate & Sensitivity & $\begin{array}{c}\text { Power } \\
\text { consumption }\end{array}$ \\
\hline [4] & Hybrid & $400-530 \mathrm{MHz}$ & BPSK & $2 \mathrm{Mbps}$ & $-13.5 \mathrm{dBm}^{\mathrm{a}}$ & N/A \\
\hline [4] & $\begin{array}{l}\text { Multichip module and } 0.35 \mu \mathrm{m} \text { CMOS } \\
\text { for ILO }\end{array}$ & $1.8-2.2 \mathrm{GHz}$ & BPSK & $2 \mathrm{Mbps}$ & $-13.5 \mathrm{dBm}^{\mathrm{a}}$ & N/A \\
\hline [5] & $90 \mathrm{~nm}$ CMOS & $\begin{array}{c}300 \mathrm{MHz} \\
868 / 915 \mathrm{MHz}\end{array}$ & BPSK & $\begin{array}{l}1 \mathrm{Mbps} \\
2 \mathrm{Mbps}\end{array}$ & $\begin{array}{l}-34 \mathrm{dBm} \\
-50 \mathrm{dBm}\end{array}$ & $\begin{array}{l}120 \mu \mathrm{W} \\
216 \mu \mathrm{W}\end{array}$ \\
\hline [22] & $65 \mathrm{~nm} \mathrm{CMOS}$ & $750-900 \mathrm{MHz}$ & BPSK & $5 \mathrm{Mbps}$ & $-43 \mathrm{dBm}^{\mathrm{b}}$ & $228 \mu \mathrm{W}$ \\
\hline [23] & Hybrid & $250-300 \mathrm{MHz}$ & FSK & $2 \mathrm{kbps}$ & N/A & $3 \mathrm{~mW}$ \\
\hline [24] & $0.18 \mu \mathrm{m}$ CMOS & $920 \mathrm{MHz}$ & FSK & $5 \mathrm{Mbps}$ & $-73 \mathrm{dBm}$ & $420 \mu \mathrm{W}$ \\
\hline This work & Hybrid and $0.18 \mu \mathrm{m}$ CMOS for ILO & $2.36-2.5 \mathrm{GHz}$ & $\begin{array}{c}\text { QPSK, } \\
\pi / 4 \text { DQPSK }\end{array}$ & 2 Msps & $\begin{array}{l}-55 \mathrm{dBm}^{\mathrm{c}} \\
-55 \mathrm{dBm}^{\mathrm{d}}\end{array}$ & $18 \mathrm{~mW}^{\mathrm{e}}$ \\
\hline
\end{tabular}

${ }^{a}$ The lowest injection power for a correct BPSK to ASK conversion

b At BER $=10^{-1}$

c At $\mathrm{EVM}=17.9 \%$

d At $\mathrm{EVM}=10.2 \%$

${ }^{\mathrm{e}}$ Including two ILOs

computed results under strong injection are not as accurate as those under weak injection. Nevertheless, the simulation can be regarded as providing an estimate of the performance of the proposed polar receiver. To sum up, the minimum injection power level for locking the oscillator of the first stage determines the sensitivity of the polar receiver, and the sensitivity can be considerably improved by inserting a low-noise amplifier (LNA) prior to this oscillator. A higher injection power corresponds to a severer AM-FM effect and consequently more degraded demodulation quality. Therefore, a mechanism for bypassing over-level received signals is suggested for this receiver. A comparison of recent ILO-based receivers [4], [5], [22]-[24] is presented in Table I. The proposed polar receiver is favorable in terms of complex modulation schemes.

\section{CONCLUSION}

This paper presents a cognitive polar receiver that utilizes two ILO stages. From a characterization of an ILO under nonconstant envelope modulation signal injection, the operating principles of the receiver, including spectrum sensing and polar demodulation, were derived in detail. The experiments verify the feasibility of the novel architecture and are mostly consistent with the theoretical predictions. Although the distortion mechanisms limit the performance of the current prototype, it suffices for short-range wireless communications. Owing to its simplicity, the proposed polar receiver has great potential for use in low-power and energy-efficient architectures. Furthermore, its spectrum sensing ability makes it more effective in cognitive radio applications.

\section{REFERENCES}

[1] W. Tuttlebee, S. Fletcher, D. Lister, T. O'Farrell, and J. Thompson, "Saving the planet-The rationale, realities and research of green radio," J. Inst. Telecommun. Professionals, vol. 4, pt. 3, pp. 8-20, Sep. 2010. [Online]. Available: http://www.theitp.org/Journal/sep_10_pdfs/ vol4_p3_8-20.pdf

[2] J. P. Costas, "Synchronous communications," Proc. IRE, vol. 47, no. 12, pp. 2058-2068, Dec. 1959.

[3] J. M. Lopez-Villegas and J. J. S. Cordoba, "BPSK to ASK signal conversion using injection-locked oscillators-Part I: Theory," IEEE Trans. Microw. Theory Tech., vol. 53, no. 12, pp. 3757-3766, Dec. 2005.
[4] J. M. Lopez-Villegas, J. G. Macias-Montero, J. A. Osorio, J. Cabanillas, N. Vidal, and J. Samitier, "BPSK to ASK signal conversion using injection-locked oscillators-Part II: Experiment," IEEE Trans. Microw. Theory Tech., vol. 54, no. 1, pp. 226-234, Jan. 2006.

[5] H. Yan, J. G. Macias-Montero, A. Akhnoukh, L. C. N. de Vreede, J. R. Long, and J. N. Burghartz, "An ultra-low-power BPSK receiver and demodulator based on injection-locked oscillators," IEEE Trans. Microw. Theory Tech., vol. 59, no. 5, pp. 1339-1349, May 2011.

[6] M. A. Tarar and Z. Chen, "A direct down-conversion receiver for coherent extraction of digital baseband signals using the injection locked oscillators," in Proc. IEEE Radio Wireless Symp., Orlando, FL, Jan. 2008, pp. 57-60.

[7] C.-T. Chen, C.-H. Hsiao, T.-S. Horng, and K.-C. Peng, "Wireless polar receiver using two injection-locked oscillator stages," in IEEE MTT-S Int. Microw. Symp. Dig., Baltimore, MD, Jun. 2011, pp. 1-4, Session WE1D-3.

[8] R. Adler, "A study of locking phenomena in oscillators," Proc. IRE, vol. 34, no. 6, pp. 351-357, Jun. 1946.

[9] L. J. Paciorek, "Injection locking of oscillators," Proc. IEEE, vol. 53, no. 11, pp. 1723-1727, Nov. 1965.

[10] R. D. Huntoon and A. Weiss, "Synchronization of oscillators," Proc. IRE, vol. 35, no. 12, pp. 1415-1423, Dec. 1947.

[11] R. C. Mackey, "Injection locking of klystron oscillators," IRE Trans. Microw. Theory Tech., vol. MTT-10, no. 4, pp. 228-235, Jul. 1962.

[12] E. F. Calandra and A. M. Sommariva, "Stability analysis of injection-locked oscillators in their fundamental mode of operation," IEEE Trans. Microw. Theory Tech., vol. MTT-29, no. 11, pp. 1137-1143, Nov. 1981

[13] B. Razavi, "A study of injection locking and pulling in oscillators," IEEE J. Solid-State Circuits, vol. 39, no. 9, pp. 1415-1424, Sep. 2004.

[14] A. Mirzaei, M. E. Heidari, and A. A. Abidi, "Analysis of oscillators locked by large injection signals: Generalized Adler's equation and geometrical interpretation," in Proc. IEEE Custom Integr. Circuits Conf., San Jose, CA, 2006, pp. 737-740.

[15] C.-J. Li, F.-K. Wang, T.-S. Horng, and K.-C. Peng, "A novel RF sensing circuit using injection locking and frequency demodulation for cognitive radio applications," IEEE Trans. Microw. Theory Tech., vol. 57, no. 12, pp. 3143-3152, Dec. 2009.

[16] G. D. Vendelin, A. M. Pavio, and U. L. Rohde, Microwave Circuit Design Using Linear and Nonlinear Techniques. New York: Wiley, 1990 , ch. 6.

[17] U. M. Ascher and L. R. Petzold, Computer Methods for Ordinary Differential Equations and Differential-Algebraic Equations. Philadelphia, PA: SIAM, 1998.

[18] H. L. Stover and R. C. Shaw, "Injection-locked oscillators as amplifiers for angle-modulated signals," in IEEE MTT-S Int. Microw. Symp. Dig., 1966, pp. 60-66.

[19] N. Lanka, S. Patnaik, and R. Harjani, "Understanding the transient behavior of injection locked LC oscillators," in Proc. IEEE Custom Integr. Circuits Conf., San Jose, CA, 2007, pp. 667-670.

[20] J. G. Proakis, Digital Communications, 4th ed. New York: McGrawHill, 2000. 
[21] P. Reynaert and M. S. J. Steyaert, "A 1.75-GHz polar modulated CMOS RF power amplifier for GSM-EDGE," IEEE J. Solid-State Circuits, vol. 40, no. 12, pp. 2598-2608, Dec. 2005.

[22] Q. Zhu and Y. Xu, "A $228 \mu \mathrm{W} 750 \mathrm{MHz}$ BPSK demodulator based on injection locking," IEEE J. Solid-State Circuits, vol. 46, no. 2, pp. 416-423, Feb. 2011.

[23] J. M. Lopez-Villegas, N. Vidal, and J. G. Macias-Montero, "FSK coherent demodulation using second-harmonic injection locked oscillator," IEEE Microw. Wireless Compon. Lett., vol. 19, no. 9, pp. 578-580, Sep. 2009.

[24] J. Bae, L. Yan, and H.-J. Yoo, "A low energy injection-locked FSK transceiver with frequency-to-amplitude conversion for body sensor network," IEEE J. Solid-State Circuits, vol. 46, no. 4, pp. 928-937, Apr. 2011.

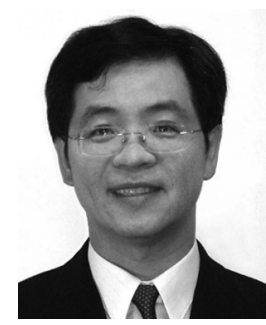

Tzyy-Sheng Horng (S'88-M'92-SM'05) was born on December 7, 1963, in Taichung, Taiwan. He received the B.S.E.E. degree from National Taiwan University, Taipei, Taiwan, in 1985, and the M.S.E.E. and Ph.D. degrees from the University of California at Los Angeles (UCLA), in 1990 and 1992, respectively.

Since August 1992, he has been with the Department of Electrical Engineering, National Sun Yat-Sen University, Kaohsiung, Taiwan, where he was the Director of the Telecommunication Research and Development Center (2003-2008) and Director of the Institute of Communications Engineering (2004-2007), and where he is currently a Professor. He has authored or coauthored over 100 technical publications published in refereed journals and conferences proceedings. He holds over ten patents. His research interests include RF and microwave integrated circuits and components, RF signal integrity for wireless system-in-package, and digitally assisted RF technologies.

Dr. Horng has served on several Technical Program Committees of international conferences including the International Association of Science and Technology for Development (IASTED) International Conference on Wireless and Optical Communications, the IEEE Region 10 International Technical Conference, the IEEE International Workshop on Electrical Design of Advanced Packaging and Systems, the Asia-Pacific Microwave Conference, the IEEE Radio and Wireless Symposium, and the Electronic Components and Technology Conference. He was the recipient of the 1996 Young Scientist Award presented by the International Union of Radio Science, the 1998 Industry-Education Cooperation Award presented by the Ministry of Education, Taiwan, and the 2010 Distinguished Electrical Engineer Award presented by the Chinese Institute of Electrical Engineering, Taiwan.

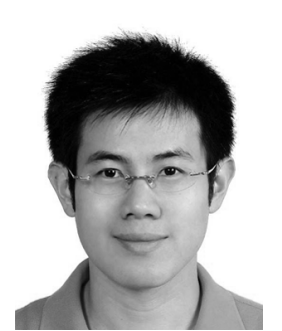

Kang-Chun Peng (S'00-M'05) was born on February 18, 1976, in Taipei, Taiwan. He received the B.S.E.E., M.S.E.E., and Ph.D. degrees from the National Sun Yat-Sen University, Kaohsiung, Taiwan, in 1998, 2000 and 2005, respectively.

He is currently an Assistant Professor with the Department of Computer and Communication Engineering, National Kaohsiung First University of Science and Technology, Kaohsiung, Taiwan. His current research interests are in the area of delta-sigma modulation techniques, low-noise PLLs, low-power VCOs, and modulated frequency synthesizers. $\mathrm{Ph} . \mathrm{D}$. degree in electrical engineering at National Sun Yat-Sen University.

His current research interests include RF power amplifiers (PAs), highly efficient and linear transmitter design, and low-power transceivers.

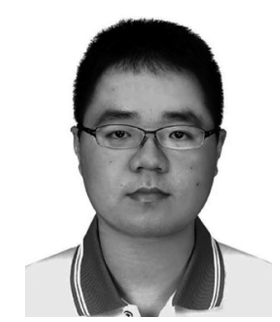

Chieh-Hsun Hsiao (S'10) was born on June 5, 1984, in Kaohsiung, Taiwan. He received the B.S.E.E. and M.S.E.E. degrees from the National Sun Yat-sen University, Kaohsiung, Taiwan, in 2006 and 2008, respectively, and is currently working toward the $\mathrm{Ph} . \mathrm{D}$. degree in electrical engineering at National Sun Yat-sen University. His doctoral research concerned PLL design and local oscillator pulling phenomena analyzing.

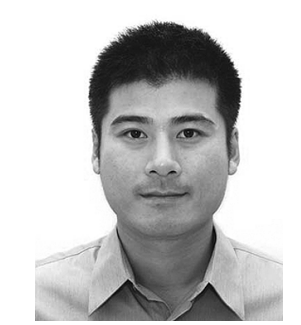

Chien-Jung Li (S'07-M'10) was born in Tainan, Taiwan, on October 26, 1979. He received the B.S.E.E. and Ph.D. degrees from National Sun Yat-Sen University, Kaohsiung, Taiwan, in 2002 and 2009, respectively.

In 2009, he was a Postdoctoral Fellow with the Department of Electrical Engineering, National Sun Yat-Sen University. Following his postdoctoral position, he joined MediaTek Inc., Hsinchu, Taiwan, as a Senior Engineer, in 2010. He is currently an Assistant Professor with the Department of Electronic Engineering, National Taipei University of Technology, Taipei, Taiwan. His research interests include power amplifier (PA) linearization techniques, frequency synthesizer designs, RF sensing circuits, injection-locking techniques, and LO pulling issues in direct-conversion transceivers. 